\title{
Influence du mode de conditionnement d'un fourrage vert sur sa composition chimique, la digestibilité in vitro de la matière organique et la dégradabilité in sacco de l'azote dans le rumen
}

\author{
M Kamoun, A Thewis \\ Faculté des Sciences Agronomiques, Unité de Zootechnie, \\ Passage des Déportés, 2 B-5800 Gembloux, Belgique
}

\begin{abstract}
Summary - Fiber contents of oven-dried ray-grass cut at different stages of maturity were higher than the values obtained for freeze-dried samples. The reverse was observed for in vitro organic matter digestibility. Moreover, oven-drying dramatically decreased in sacco nitrogen degradability as compared to lyophilization, preservation by freezing with liquid nitrogen and use of fresh material.
\end{abstract}

Introduction - Les estimations de la composition chimique, la valeur nutritive et la dégradabilité ruminale des matières azotées des fourrages verts peuvent être fortement influencées par le mode de conditionnement des échantillons (Abdalla et al, 1988; Ould-Bah, 1989). On note cependant certaines contradictions entre les différents auteurs.

Notre objectif est d'une part de comparer l'influence du mode de séchage sur la composition chimique et la digestibilité in vitro de la matière organique d'un raygrass italien récolté à deux stades végétatifs, d'autre part d'étudier l'effet de quatre modes de conditionnement de ce même fourrage sur la dégradabilité in sacco de l'azote dans le rumen.

Matériel et Méthodes - Un ray-grass italien récolté à deux stades de végétation (début épiaison et repousse d'arrière-saison) est haché en brins de 4 à $10 \mathrm{~mm}$. Après mélange, la masse est divisée en quatre parties subissant chacune un des traitements suivants : utilisation à l'état frais (F), conservation sous azote liquide pendant $48 \mathrm{~h}$ (NI), congélation à $-20^{\circ} \mathrm{C}$ puis lyophilisation (L) et séchage pendant $48 \mathrm{~h}$ à l'étuve ventilée à $60^{\circ} \mathrm{C}(\mathrm{E})$. Les échantillons séchés à l'étuve et lyophilisés sont broyés à la grille de 1 $\mathrm{mm}$ puis analysés pour leur teneur en fibres (NDF et ADF) et en matières azotées totales (MAT). La digestibilité in vitro de la matière organique (dMO) est également déterminée sujvant la méthode enzymatique décrite par Vanderhaeghe et Biston (1987). En vue de suivre la cinétique de dégradation de l'azote, des sachets de nylon (dimensions : $20 \times 10 \mathrm{~cm}$; pores : $42,5 \mu \mathrm{m} ; 2$ sachets par point de cinétique) contenant l'équivalent d'environ 2,5 et $4 \mathrm{~g}$ de MS (en brins de 4 à $10 \mathrm{~mm}$ ) pour la repousse et le fourrage récolté début épiaison respectivement, sont incubés successivement dans le rumen de 3 moutons pendant $3,6,12$, 24 et $48 \mathrm{~h}$ suivant la séquence : $F$ ( $3 \mathrm{~h}$ après la récolte), NI ( $2 \mathrm{~h}$ après décongélation), $\mathrm{E}$ et $\mathrm{L}$. La dégradabilité de l'azote du fourrage est déterminée selon la méthode de Ørskov et McDonald (1979). Afin de permettre la comparaison entre traitements pour un stade de végétation donné (analyse de la variance à deux critères de classification suivi d'un test de Newman et Keuls), un témoin (foin de prairie broyé) est introduit dans chaque série.

Résultats et Discussion - Le séchage à l'étuve à $60^{\circ} \mathrm{C}$ entraîne par rapport à la lyophilisation des teneurs en fibres plus élevées et une digestibilité in vitro de la matière organique plus faible (tableau I). 
Tableau I. Influence du mode de conditionnement (C) d'un ray-grass italien récolté à deux stades de végétation sur la composition chimique (en \% de la MS), la digestibilité in vitro de la matière organique (dMO) et la dégradabilité in sacco de l'azote dans le rumen (DT pour $k=0,06)(\bar{x} \pm \sigma)$.

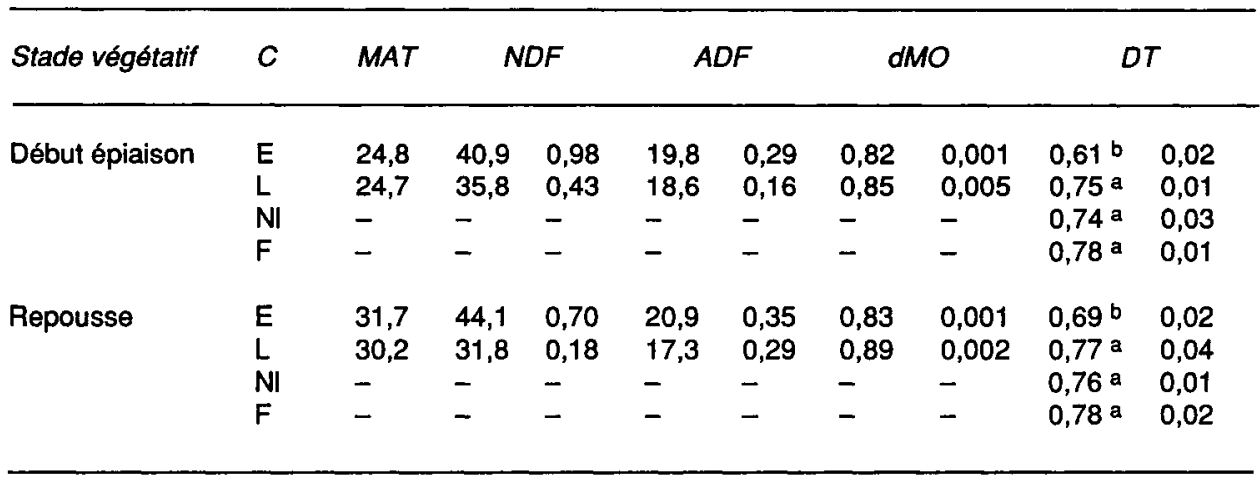

a, b Dans une même colonne et pour chaque stade, les valeurs affectées d'une lettre différente sont significativement différentes $(P<0,05)$.

L'écart entre les deux modes de séchage paraît d'autant plus marqué que le fourrage est jeune. Des quatre modes de conditionnement envisagés, seul le séchage à l'étuve diminue, par rapport au fourrage vert frais, la dégradabilité in sacco des matières azotées. Ces observations peuvent s'expliquer par la formation de ponts entre les chaînes polypeptidiques et de "composés de Maillard". Dans notre cas, la congélation n'affecte pas significativement la dégradabilité de l'azote : ce traitement n'aurait donc pas provoqué un éclatement significatif des cellules végétales.

En conclusion, la lyophilisation apparaît comme la seule méthode de conservation pratique et recommandable en vue de l'analyse des fourrages verts. Les valeurs de DT sont toutefois inférieures aux valeurs in vivo mentionnées dans la littérature, vraisemblablement à cause de la coIonisation microbienne.

Remerciements - Ce travail a été financé par le Fonds de la Recherche Fondamentale Collective (FRFC, convention 2.4542.84).

Abdalla HO, Fox DG, van Soest PJ (1988) $J$ Anim Sci 66, 2646-2649

Ørskov ER, McDonald I (1979) J Agric Sci 92, 499-503

Ould-Bah MY (1989) Thèse, Université des Sciences et Techniques du Languedoc, Montpellier

Vanderhaeghe S, Biston R (1987) Bull Rech Agron Gembloux 22, 209-219 